\title{
LETTERS
}

\section{The authors respond to "Fentanyl patch probably not related to amnesia in case"}

We thank Dr. Romayne Gallagher for her letter to the editor ${ }^{1}$ regarding our article published in CMAJ, which describes a case of bilateral hippocampal injury and amnesia after pharmaceutical fentanyl overdose. ${ }^{2}$

Regarding concerns about the potential for fentanyl to have produced overdose in this case, Dr. Gallagher writes,

... the time from application [of fentanyl patch] to ... maximum serum concentration is 12 to 48 hours. ... The patient described in this case was found "unresponsive" 12 hours after the patches were initiated. ${ }^{1}$

The patient described in our case report was found 12-13 hours after applying fentanyl patches, which is within the time window to reach maximum serum concentration at steady state reported above. The window reported by Dr. Gallagher is also longer than what is described in the article by Muijsers and Wagstaff cited in Dr. Gallagher's letter (12-24 hr). ${ }^{3}$

Dr. Gallagher also states,

When initiating the fentanyl patch, the current opioid is continued for 12 hours to provide sufficient analgesia and to prevent withdrawal symptoms. ${ }^{1}$

The morphine equivalent dose of transdermal fentanyl $37 \mu \mathrm{g} / \mathrm{h}$ is $135-$ 179 mg every 24 hours in some monographs. The morphine equivalent dose of hydromorphone controlled release (CR) $9 \mathrm{mg} 3$ times per day (TID) is $135 \mathrm{mg}$ every 24 hours. Concerningly, the patient's hydromorphone dose was the lowest possible equivalent to $37 \mu \mathrm{g} / \mathrm{h}$ of fentanyl. Conversely, a local pain specialist and most sources recommend using half the equivalent dose of a new opioid initially when switching to avoid overdose from incomplete cross-tolerance. ${ }^{4}$

To address Dr. Gallagher's response regarding cross-tolerance, we contacted the family to confirm adherence to the patient's previously prescribed medica- tions. We learned that she had not been taking hydromorphone $9 \mathrm{mg}$ CR orally TID as prescribed in the months before her overdose. This was confirmed with her pharmacy, where a 1-month supply of hydromorphone CR $9 \mathrm{mg}$ TID (90 tabs) was filled several months before her presentation to hospital. As such, the effect of naloxone in this case was probably that it reversed fentanyl toxicity, rather than hydromorphone as was suggested by Dr. Gallagher. ${ }^{1,2}$ Although this point is regrettably overlooked in the original manuscript, it strengthens the implication of fentanyl's role in the amnestic syndrome.

We maintain that the fentanyl exposure in the case is sufficient to potentially induce respiratory depression and decreased level of consciousness from incomplete cross-tolerance. Furthermore, lowered opioid tolerance because of nonadherence to prescribed opioids substantially increases the probability of fentanyl overdose. Notably, most sources advise against fentanyl use in opioid-naive patients.

However, it is not clear whether severe clinical overdose, hypoxemia or hypotension are required or sufficient for hippocampal injury to occur in opioidassociated amnestic syndrome. Only 5 of 16 cases reported to date had severe overdose requiring invasive airway management. ${ }^{5}$ In at least 1 case, a patient presented to the emergency department with amnesia after using fentanyl but was not treated for overdose. ${ }^{6}$

Regarding previous cases of opioidassociated amnestic syndrome, Dr. Gallagher writes,

In the report of 13 cases, all patients had a history of substance use, and some tested positive for other prescription medications or illicit drugs. ${ }^{1}$

The potential role of adulterants, toxins or other agents in opioid-associated amnestic syndrome was acknowledged in the present and previous publications. . $^{2,5-8}$ In previous cases, some patients did test positive for other substances; however, among those who did not are patients who tested positive for fentanyl only ${ }^{7}$ or who recently had used only fentanyl. ${ }^{6}$ There was no exposure to other toxins in the present case.

Dr. Gallagher also states,

The 3 cases that potentially involved prescription fentanyl all involved anesthesia in which multiple drugs were used. One episode was reversed with flumazenil, and midazolam was suggested as the possible cause. ${ }^{1}$

In cited cases of postanesthesia transient global amnesia, all patients received pharmaceutical fentanyl in addition to other medications. Given that amnesia was self-limited in these cases, we do not feel that it is certain that the 1 patient who received flumazenil improved because of the reversal agent; it is possible that recovery would have occurred spontaneously. This syndrome may be distinct from opioid-associated amnestic syndrome given its transient nature, although further study to determine potential mechanistic similarities is warranted.

Regarding animal models of opioid neurotoxicity to limbic structures, Dr. Gallagher states,

As Taylor and colleagues note, only in rats has fentanyl been shown to exert a direct effect on the hippocampus. ${ }^{1}$

Dr. Gallagher is correct that only in rodent models has fentanyl been shown to have a direct toxic effect on limbic structures (see Dutton ${ }^{8}$ and Butler and colleagues ${ }^{6}$ for further citation of relevant literature). Although the limitations of animal models in clinical research is widely recognized in the medical community, the potential harm of fentanyl remains concerning and, as such, this literature is worth acknowledging despite inherent limitations.

Finally, regarding key points and conclusions drawn in our article about the role of fentanyl in opioid-associated amnestic syndrome, Dr. Gallagher writes,

Readers who just read the article title and key points will be misled, as the key points state, "[f]entanyl, a synthetic opioid, has been implicated in opioid-associated amnestic syndrome in cases of prescription and nonprescription opioid use ...". This statement is not accurate. ${ }^{1}$ 
Fentanyl has been demonstrably implicated (i.e., brought into intimate or incriminating connection) in opioidassociated amnestic syndrome in cases of prescription and nonprescription opioid use, including several nonprescription cases in which it was either the only drug detected, ${ }^{7}$ or the only drug used in recent history. ${ }^{6}$

$\mu$-Opioid receptors are found in the hippocampi. ${ }^{9}$ Furthermore, the endogenous opioid system plays an important role in limbic function in various clinical contexts related to memory. ${ }^{10-12}$ Therefore, it is possible that opioid toxicity could precipitate toxic-metabolic failure of limbic structures, leading to amnesia. Limbic toxicity has been shown in animal models of opioid overdose.

Opioid-associated amnestic syndrome is rare and does not occur in every patient who takes opioids. Published cases suggest the presence of some unidentified contributory factor. However, we find it especially concerning that opioid-associated amnestic syndrome has emerged at the peak of an opioid crisis being driven in large part by accidental ingestion of fentanyl. ${ }^{13}$ This concern, raised in earlier versions of our manuscript, preceded a chain of events that led to the detection of synthetic opioids in the original cohort described by Barash and colleagues. $^{7}$

At the time of a recent telephone call with the patient's family, the patient continued to experience substantial residual memory impairment. Since our article was published, persistent morphological changes have been identified in the hippocampus of another patient with fentanyl overdose. ${ }^{6}$

Opioid-associated amnestic syndrome is a controversial entity. ${ }^{1,8}$ However, what has emerged is a distinct syndrome with acute and persistent physiological and structural changes to the limbic system leading to devastating cognitive impairment after opioid use. Further exploration of a potential link with pharmaceutical fentanyl is a pressing issue for policy-makers and health care professionals alike.

\section{Ryan G. Taylor MD}

Neurology resident, Department of Clinical Neurological Sciences, Western University, London, Ont.

\section{Seyed M. Mirsattari MD}

Associate professor of neurology, Department of Clinical Neurological Sciences, Western University, London, Ont.

\section{Donald H. Lee MD}

Professor, Department of Clinical Neurological Sciences and Division of Radiology, Western University, London, Ont.

- Cite as: CMAJ 2019 November 4;191: E1226-7. doi: 10.1503/cmaj.73774

\section{References}

1. Gallagher RE. Fentanyl patch probably not related to amnesia in case [letter]. CMAJ 2019; 191:E1225.

2. Taylor RG, Budhram A, Lee DH, et al. Opioidassociated amnestic syndrome observed with fentanyl patch use. CMAJ 2019;191:E337-9.

3. Muijsers RB, Wagstaff AJ. Transdermal fentanyl: an updated review of its pharmacological prop- erties and therapeutic efficacy in chronic cancer pain control. Drugs 2001;61:2289-307.

4. Kahan M, Wilson L, Mailis-Gagnon A, et al.; National Opioid Use Guideline Group. Canadian guideline for safe and effective use of opioids for chronic noncancer pain: clinical summary for family physicians. Part 2: special populations. Can Fam Physician 2011;57:1269-76, e419-28.

5. Barash JA, Somerville N, DeMaria A Jr. Cluster of an unusual amnestic syndrome - Massachusetts, 2012-2016. MMWR Morb Mortal Wkly Rep 2017;66:76-9.

6. Butler PM, Barash JA, Casaletto KB, et al. An opioid-related amnestic syndrome with persistent effects on hippocampal structure and function. J Neuropsychiatry Clin Neurosci 2019; 31:392-6.

7. Barash JA, Ganetsky M, Boyle KL, et al. Acute amnestic syndrome associated with fentanyl overdose. N Engl J Med 2018;378:1157-8.

8. Dutton RP. More on acute amnestic syndrome associated with fentanyl overdose. N Engl J Med 2018;378:2247.

9. Drake CT, Milner TA. Mu opioid receptors are in discrete hippocampal interneuron subpopulations. Hippocampus 2002;12:119-36.

10. Burtscher J, Schwarzer C. The opioid system in temporal lobe epilepsy: functional role and therapeutic potential. Front Mol Neurosci 2017 ; 10:245.

11. Torres-Berrio A, Nava-Mesa MO. The opioid system in stress-induced memory disorders: from basic mechanisms to clinical implications in post-traumatic stress disorder and Alzheimer's disease. Prog Neuropsychopharmacol Biol Psychiatry 2019;88:327-38.

12. Thomas SA. Neuromodulatory signaling in hippocampus-dependent memory retrieval. Hippocampus 2015;25:415-31.

13. Overdose death rates. Bethesda (MD): National Institute on Drug Abuse; 2019. Available: www. drugabuse.gov/related-topics/trends-statistics/ overdose-death-rates (accessed 2019 Sept. 1).

\section{Competing interests: None declared.}

Editor's note: A correction to the case has been published (www.cmaj.ca/lookup/doi/10.1503/ cmaj.191311), and the article has been updated at cmaj.ca. 